\title{
Hemoglicoteste: influência dos locais de punção sobre os níveis de glicose e intensidade de dor
}

\author{
Hemoglucotest: influence of puncture sites on levels \\ of glucose and pain intensity
}

\author{
Ana Paula Bornhausen ${ }^{1}$, Cleiton Jonei Reginatto ${ }^{1}$, Vinicius Mazzetti Subtil ${ }^{1}$, Eduardo Mazzetti Subtil ${ }^{1}$, Lourenço Duarte Zanotto ${ }^{1}$ \\ Denise Krieger ${ }^{1}$, Everly Rosane Goetz ${ }^{1}$, Vitor Engrácia Valenti², Luiz Carlos de Abreu³ ${ }^{2}$ Fernando Henrique de Sousa ${ }^{4}$, \\ Roberto Odair Grassi', Rafael Frizon'1, Márcio Petenusso' \\ 'Universidade do Planalto Catarinense (UNIPLAC) - Lages (SC), Brasil. \\ ¿Departamento de Fonoaudiologia, Faculdade de Filosofia e Ciências, Universidade Estadual Paulista "Júlio de Mesquita Filho" (UNESP) - Marília (SP), Brasil. \\ ${ }^{3}$ Laboratório de Escrita Científica, Faculdade de Medicina do ABC (FMABC) - Santo André (SP), Brasil. \\ ${ }^{4}$ Faculdade de Ciências, UNESP - Bauru (SP), Brasil.
}

DOI: http://dx.doi.org/10.7322/abcshs.v39i3.652

\section{RESUMO}

Introdução: O monitoramento da glicose pelo portador de diabetes melito é importante na prevenção de complicações clínicas. Objetivo: Comparar a intensidade de dor nas punções em lóbulo da orelha e polpa digital e a interferência do local de punção sobre os valores do exame de glicemia capilar. Método: Estudo exploratório, descritivo e quantitativo. Participaram 70 portadores de diabetes melito. A monitorização foi realizada ao acaso, com glicosímetro G-TECH Free $1^{\circledR}$. Para avaliação da intensidade da dor, utilizou-se uma escala numérica graduada em 0 (ausente), 1 (muito leve), 2 (leve), 3 (moderada) e 4 (intensa). Resultados: Para os portadores de diabetes melito Tipo 1, a glicemia média foi de 274,38 $\pm 22,64 \mathrm{mg} / \mathrm{dL}$ para lóbulo da orelha e de $298,33 \pm 4,58 \mathrm{mg} / \mathrm{dL}$ para polpa digital. Para DM Tipo 2, os valores foram 187,19 $\pm 9,85 \mathrm{mg} / \mathrm{dL}$ para lóbulo da orelha e 195,41 10,47 mg/dL para polpa digital. Houve correlação entre as glicemias encontradas nos dois locais de punção. Para os portadores de diabetes melito Tipo 1, observou-se ausência de dor (0) em lóbulo da orelha e polpa digital com 1,86 $\pm 0,12$. Para portadores de diabetes melito Tipo 2, obteve-se que a média de intensidade de dor para lóbulo da orelha foi de 0,06 $\pm 0,03$ e para polpa digital, 1,75 $\pm 0,09$. Conclusão: Houve diferença estatística quanto ao índice glicêmico gerado pelos diferentes locais. Menor intensidade de dor foi observada quando o teste é realizado no lóbulo da orelha, sendo uma boa opção para o local de exame.

Palavras-chave: glicemia; diabetes mellitus; antissepsia; glicose.

\begin{abstract}
Introduction: Glucose self-monitoring by diabetes mellitus patients is important for the prevention of clinical complications. Objective: To compare the pain intensity in punctures at earlobe and fingertip and to evaluate the interference of the puncture site on the values of capillary blood glucose test. Method: Descriptive and exploratory quantitative study. We evaluated 70 diabetes mellitus patients. The monitoring was randomly carried out, with G-TECH Free glucometer $1^{\circledR}$. To assess pain intensity, it was used a numerical scale graded in 0 (absent), 1 (mild), 2 (mild), 3 (moderate), and 4 (severe). Results: For patients with Type 1 diabetes mellitus, the average blood glucose was $274.38 \pm 22.64 \mathrm{mg} / \mathrm{dL}$ for earlobe and $298.33 \pm 4.58 \mathrm{mg} / \mathrm{dL}$ for fingertip. For Type 2 diabetes mellitus, results were $187.19 \pm 9.85 \mathrm{mg} / \mathrm{dL}$ for earlobe and $195.41 \pm 10.47 \mathrm{mg} / \mathrm{dL}$ for fingertip. There was correlation between blood glucoses measured in both puncture sites. For patients with Type 1 diabetes mellitus, there was no pain (0) in earlobe, and fingertip was $1.86 \pm 0.12$. For patients with Type 2 diabetes mellitus, it was found that the average pain intensity for earlobe was $0.06 \pm 0.03$ and for fingertip, 1.75 \pm 0.09 . Conclusion: There was a statistical difference regarding the glycemic index generated by different sites. Lower pain intensity was observed when the test was done on the earlobe, as a good choice for test site.
\end{abstract}

Keywords: blood glucose; diabetes mellitus; antisepsis; glucose. 


\section{INTRODUÇÃO}

Os avanços da Medicina vêm proporcionando mudanças na terapêutica do diabetes melito (DM), visando melhorar a qualidade de vida dos portadores da doença ${ }^{1}$. Atualmente, entende-se que o controle adequado da DM é essencial para prevenir ou retardar as complicações crônicas de tal distúrbio metabólico, sendo o automonitoramento, por meio da glicemia capilar, um de seus alicerces ${ }^{2}$

O controle inadequado da concentração glicêmica por longos períodos associa-se a complicações como hipertensão arterial sistêmica, insuficiência renal, disfunção erétil, cetoacidose na DM, retinopatia diabética, acidente vascular encefálico, infarto agudo do miocárdio, pé diabético, vertigens, síncope e até coma hiperosmolar hiperglicêmico ${ }^{3}$.

Os métodos de automonitoramento da glicemia capilar disponíveis no mercado, recentemente, oferecem resultado imediato acerca da concentração glicêmica nos vasos capilares, por meio da fotometria de refletância ou da eletroquímica, além de marcar a data e a hora do exame ${ }^{4}$.

O automonitoramento da glicose pelo paciente é importante no auxílio da identificação de alterações glicêmicas, possibilitando a prevenção das complicações ${ }^{5}$. Locais alternativos para testes de glicemia têm sido estudados, entretanto, poucos estudos incluem alguns com o lóbulo da orelha ${ }^{6-8}$. Bina et al..$^{9}$ encontraram em um estudo de pacientes diabéticos tipo 2 que o teste de localização alternativo foi consistente com os resultados da glicemia do dedo em jejum e duas horas após refeição.

O exame em questão é realizado pela glicemia capilar ou hemoglicoteste, que é um teste quantitativo para glicose. Existem várias marcas e modelos de glicosímetro com o mesmo princípio de uso e análise bioquímica. O método para obtenção do resultado da concentração da glicose é a fotometria de refletância ou eletroquímica. No medidor eletrônico é possível marcar hora, data do exame e glicose em $\mathrm{mg} / \mathrm{dL}^{5}$.

Uma investigação anterior ${ }^{7}$ mostrou que a punção da pele do lóbulo da orelha é menos dolorosa do que a do polegar. Embora a diferença de pontuações medianas da dor seja pequena, nenhum dos pacientes do grupo de orelha experimentou elevados níveis de dores. A razão para tal diversidade não está clara; no entanto, a densidade de nociceptores pode ser menor do que na orelha e no dedo polegar. Além disso, o efeito pode ser influenciado pela percepção dos pacientes, em especial no que eles não podem ver na orelha a ser testada.

Para o teste, deve-se escolher o local da punção, em seguida introduzir a lanceta no dispositivo ajustando para locais mais profundos ou com grande calosidade, a profundidade de número maior; em áreas menos profundas, a de número menor. Após a punção, observa-se que a formação da gota de sangue encosta-se na fita do teste colocada no medidor até preencher a área indicada. Esta é geralmente impregnada de glicose-oxidase, ou seja, a enzima específica da glicose e com reativo de cor, a qual possibilita que o resultado apareça no visor. $\mathrm{O}$ teste deve ser realizado antes ou duas horas após as refeições, ou após a injeção de insulina, para aqueles que a utilizam ${ }^{10}$.

Um dos aspectos que dificultam a realização do exame é a dor, visto que o local mais utilizado para realizá-lo é nas falanges distais dos dedos da mão, tornando-se um dos fatores limitantes para o automonitoramento. Repetidas punções causam desconforto e aparecimento de calosidades no local ${ }^{10}$. Esta região contém inúmeras terminações nervosas, pois na face palmar das falanges o número dos corpúsculos de Meissner e receptores de Merkel existem em maior quantidade, explicando a sensibilidade, que provoca elevada sensação dolorosa nas punções ${ }^{11,12}$.

Regiões alternativas para a realização deste teste são: antebraços, palma da mão, panturrilha e lóbulo da orelha. No Brasil, há poucos estudos a respeito do lóbulo da orelha como opção ao teste. Portanto, este estudo procurou comparar a intensidade de dor nas punções em lóbulo da orelha e polpa digital e se existe interferência do local de aplicação sobre os valores glicêmicos obtidos.

\section{MÉTODOS}

Tratou-se de uma pesquisa exploratória, descritiva de abordagem quantitativa, com paciente diabético tipos 1 e 2 na cidade de Lages, Santa Catarina. O estudo foi aprovado pelo Comitê de Ética em Pesquisa sob o n ${ }^{\circ}$ 037-12. Foram avaliados 70 pacientes maiores de 18 anos que concordaram e assinaram um termo de consentimento livre e esclarecido.

A monitorização foi realizada pelo pesquisador, ao acaso, em uma Unidade de Saúde da Família, com glicosímetro G-TECH Free $1^{\circledR}$, apresentando Registro Agência Nacional Vigilância Sanitária Ministério da Saúde: 80275310033. Fitas-teste que acompanham o aparelho foram utilizadas para verificar sua acurácia. Padronizou-se o uso do grau médio $(4 \mathrm{~mm})$ da lanceta para todos os exames. Realizou-se antissepsia com álcool etílico a $70 \%$. Esperou-se 10 segundos para o tempo de ação e evaporação do álcool, e então puncionou-se a face ventral da falange distal do terceiro dedo da mão direita. A punção do lóbulo inferior da orelha ocorreu simultaneamente.

A avaliação da intensidade da dor foi baseada em escala numérica graduada em 0 (ausente), 1 (muito leve), 2 (leve), 3 (moderada) e 4 (intensa) $)^{13}$, proposta no estudo de Ferraz, Maia e Araújo $^{13}$. Os resultados foram expressos em $\mathrm{mg} / \mathrm{dL}$ de sangue. Foram excluídos deste estudo os pacientes com neuropatia diabética preestabelecida; com amputação do terceiro dedo da mão direita; que não possuíam a orelha direita; que estavam em uso de analgésicos potentes ou anticoagulantes e que apresentavam discrasias sanguíneas.

Os dados foram registrados em ficha padrão e analisados por média ponderada da análise de gráficos e tabelas, por meio do teste estatístico $t$ de Student. Para analisar a correlação entre a glicemia por meio da polpa digital e do lóbulo da orelha, aplicou-se o teste de correlação de Pearson. Foi considerada correlação forte para $r>0,75$, moderada para r entre 0,75 e 0,5 e 
fraca para $r<0,5$. Utilizou-se o pacote Biostat 2009, versão 5.8 .4 para Windows 7. Valores de significância foram considerados para $\mathrm{p}<0,05$

\section{RESULTADOS}

O estudo foi realizado no período de agosto a setembro de 2012. Dos 70 pacientes participantes, 25 (35,71\%) eram homens

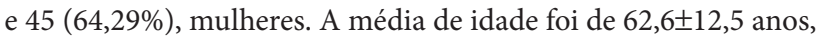
sendo a mínima de 17 e a máxima de 89 anos. O intervalo de maior prevalência foi o de $56(7,4 \%)$ e 64 anos $(7,4 \%)$.

A porcentagem dos portadores de DM Tipo 1 (DM1) foi de 30 (21) e de DM Tipo 2 (DM2), 70 (49). Houve um total de 37,1\% de sujeitos que diagnosticaram a doença há menos de um ano e $11,4 \%$ possuem a doença há pelo menos 10 anos.

A Tabela 1 mostra que os portadores de DM1 obtiveram média de 274,38 $\pm 22,64 \mathrm{mg} / \mathrm{dL}$ para o índice glicêmico em lóbulo da orelha, e de $298,33 \pm 4,58 \mathrm{mg} / \mathrm{dL}$ para polpa digital. Observa-se na Tabela 1 que os portadores de DM2 tiveram média do índice glicêmico para lóbulo da orelha de $187,19 \pm 9,85 \mathrm{mg} / \mathrm{dL}$ e para polpa digital de 195,41 $\pm 10,47 \mathrm{mg} / \mathrm{dL}$. As análises dos dados pelos testes estatísticos apontam diferença estatística significante da glicemia capilar entre os locais analisados.

Em relação à dor, a Tabela 1 mostra que os portadores de DM1 conseguiram uma média de $0,0 \pm 0,0$ para o índice de dor no lóbulo da orelha, e em polpa digital de 1,86 $\pm 0,12$.

Além disso, para portadores de DM2, obteve-se a média do índice de dor para lóbulo da orelha de $0,0 \pm 0,03$ e para polpa digital de $1,75 \pm 0,09$. O teste estatístico mostra diferença significativa entre os locais analisados. Quanto à região do hemoglicoteste, verificou-se que os portadores de DM1 e DM2 tiveram maior satisfação para punção em lóbulo da orelha, tendo menor sensação dolorosa neste local.

Houve correlação forte e significante entre a glicemia por meio da polpa digital e do lóbulo da orelha $(r=0,948 ; \mathrm{p}<0,0001)$, como visto na Figura 1.

\section{DISCUSSÃO}

No Brasil, a incidência de complicações crônicas da DM vem aumentando, o que pode ter relação com a ausência de programas que capacitem profissionais de saúde e pacientes de forma efetiva.

Tabela 1: Glicemia e análise comparativa do índice de dor no lóbulo da orelha e na polpa digital. Escala numérica para a dor graduada em 0 (ausente), 1 (muito leve), 2 (leve), 3 (moderada) e 4 (intensa)

\begin{tabular}{|c|c|c|c|c|}
\hline & & $\begin{array}{c}\text { Lóbulo da } \\
\text { orelha }\end{array}$ & Polpa digital & Valor $p$ \\
\hline \multirow{2}{*}{ Glicemia } & DM1 & $274,38 \pm 34 \mathrm{mg} / \mathrm{dL}$ & $298,33 \pm 43 \mathrm{mg} / \mathrm{dL}$ & $<0,05$ \\
\hline & DM2 & $187,19 \pm 22 \mathrm{mg} / \mathrm{dL}$ & $195,41 \pm 38 \mathrm{mg} / \mathrm{dL}$ & $<0,05$ \\
\hline \multirow{2}{*}{ Índice de dor } & DM1 & $0 \pm 0$ & $1,86 \pm 0,6$ & $<0,05$ \\
\hline & DM2 & $0,06 \pm 0,01$ & $1,75 \pm 1$ & $<0,05$ \\
\hline
\end{tabular}

O hemoglicoteste é essencial para portadores de DM, sendo realizado geralmente em polpa digital. Por isso, a dor gerada faz com que os pacientes não façam uma monitorização adequada. A realização do hemoglicoteste em locais alternativos tem acurácia semelhante à da polpa digital. Ferraz, Maia e Araújo ${ }^{13}$ analisaram simultaneamente o índice glicêmico em lóbulo da orelha e polpa digital de 40 pacientes com DM2, não havendo diferença estatística significativa entre os testes realizados. Viana et al. ${ }^{14}$ avaliaram também simultaneamente o índice glicêmico de polpa digital, antebraço, braço, coxa, panturrilha, região tenar e hipotenar da mão direita em 100 pacientes com DM, concluindo a eficácia da glicemia gerada por locais alternativos, já que não houve diferença estatística significante. A Tabela 1 mostra que nosso estudo não corrobora tais pesquisas, uma vez que foi encontrada uma diferença estatística significativa entre os níveis glicêmicos em lóbulo da orelha e polpa digital. O índice glicêmico para polpa digital estava superior àquele para lóbulo da orelha, em ambos os grupos de pacientes, demonstrando que o local da punção pode interferir nos resultados de glicemia capilar. No entanto, não há nenhum aspecto anatomofisiológico que justifique este dado.

De acordo com a Tabela 1, o lóbulo da orelha foi o local com o menor índice de sensação dolorosa. Dor é uma sensação desagradável originada por algum estímulo, em resposta protetora e adaptativa para o organismo, que pode causar dano aos tecidos. Os receptores cutâneos para a dor são denominados nociceptores, os quais são ativados por algum estímulo mecânico, térmico ou químico. Além desses, há os mecanonociceptores que respondem somente a estímulos mecânicos intensos, não respondendo aos químicos ou térmicos a menos que sejam sensibilizados previamente. Os mecanociceptores estão distribuídos na superfície da pele e no subcutâneo, sendo um receptor de adaptação rápida. Os tipos são os corpúsculos de Merkel, Meisnner, Pacini e Ruffini. Todos esses receptores são inervados por terminações de fibras mielínicas grossas do tipo A beta. A distribuição na mão segue um padrão e, na face

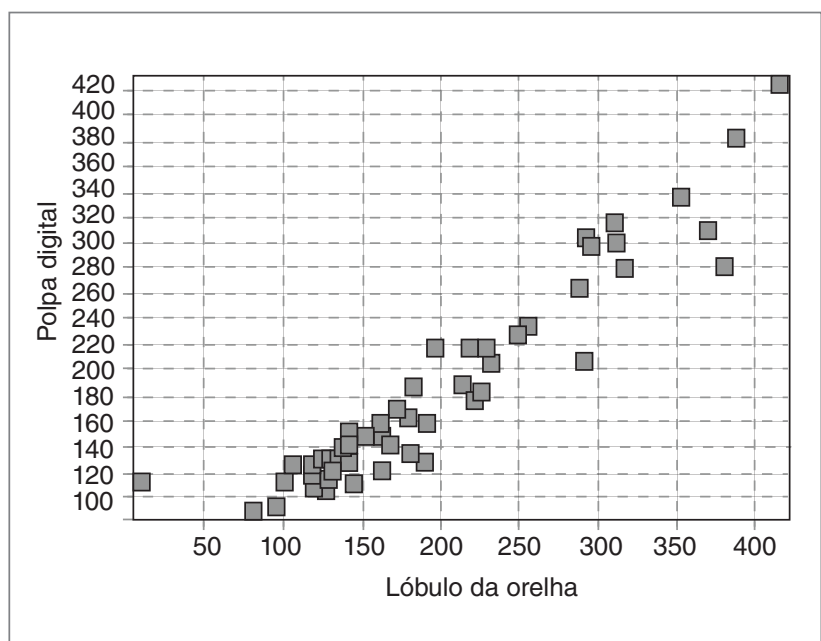

Figura 1: Coeficiente de correlação entre a glicemia por meio da polpa digital e do lóbulo da orelha $(r=0,948 ; p<0,0001)$ 
palmar das falanges, o número dos corpúsculos de Meisnner e receptores de Merkel é mais alto, explicando assim a grande sensibilidade que existe nesta área. Tal fato dificulta os hemoglicotestes em função da sensação dolorosa ser muito alta, o que é explicado pelo homúnculo de Penfield e Rasmussen, no qual as áreas com maior densidade de receptores cutâneos para a dor são ponta dos dedos da mão, lábios e língua ${ }^{12}$.

Conforme nossos dados, o método para monitoramento de glicemia por meio do lóbulo da orelha pode ser sugerido como uma alternativa. Um estudo anterior ${ }^{8}$ analisou medições glicêmicas do lóbulo da orelha, e os autores observaram que o coeficiente de correlação entre o método empregado e a coleta realizada na ponta do dedo foi de $0,97(\mathrm{p}<0,01)$. Deste modo, nossos dados e o estudo mencionado suportam o monitoramento de glicemia por meio do lóbulo da orelha.

Para o índice doloroso superior em DM1, uma explicação seria a possível neuropatia sensitiva periférica que é comum neste grupo, causada por alterações no sistema nervoso periférico e autonômico que está também ligada ao tempo de diabetes e à idade do paciente.
Há um crescente interesse em locais menos dolorosos alternativos para monitoramento de glicemia. A ponta do lóbulo da orelha é um local em potencial, que tem sido usada ocasionalmente por enfermeiros quando o teste do dedo é recusado ou está difícil ${ }^{6-8}$. Para muitos pacientes, a técnica de monitoramento pelo lóbulo pode ser menos conveniente porque necessita de uma segunda pessoa para recolher o sangue ${ }^{7}$. Isso, certamente, limita a aplicabilidade da técnica em ambiente ambulatorial. Porém, testes de orelha parecem ser uma alternativa útil que poderia minimizar custos e desconforto em pacientes atendidos por um parente ou enfermeira em hospitais e asilos.

Nosso estudo demonstrou que, quando a punção foi realizada no lóbulo da orelha, houve menor intensidade de dor, o que leva a crer que este local consiste numa alternativa para a realização do teste, podendo os pacientes serem estimulados a realizá-lo em frente ao espelho. No entanto, os dados demonstraram que houve diferença estatística quanto ao índice glicêmico gerado pelos diferentes locais, devendo estas informações serem comprovadas por estudos com maior número de hemoglicotestes no mesmo paciente, por um maior período.

\section{REFERÊNCIAS}

1. Collet-Solberg PF. Cetoacidose diabética em crianças: revisão da fisiopatologia e tratamento com o uso do "método de duas soluções salinas". J Pediatr. 2001;77(1):9-16. http://dx.doi.org/10.1590/S0021-75572001000100006

2. Burge MR. Lack of compliance with home blood glucose monitoring predicts hospitalization in diabetes. Diabetes Care. 2001;24(8):1502-3.

3. Sociedade Brasileira de Diabetes [Internet]. Diretrizes SDB 2009. Disponivel em: http://www.diabetes.org.br/para-profissionais/ diretrizes-da-sbd. Acesso em: 10 set. 2012.

4. Jacobson AM, Groot M, Samson JA. The evaluation of two measures of quality of life in patients with type 1 and type 2 diabetes. Diabetes Care. 1994;17(4):267-74

5. Ravel R. Laboratório clínico: aplicações clínicas dos dados laboratoriais. Rio de Janeiro: Guanabara Koogan; 1995.

6. Anzalone P. Equivalence of earlobe site blood glucose testing with finger stick. Clin Nurs Res. 2008;17(4):251-61. http://dx.doi.org/10.1177/1054773808325050

7. Carley SD, Libetta C, Flavin B, Butler J, Tong N, Sammy I. An open prospective randomised trial to reduce the pain of blood glucose testing: ear versus thumb. BMJ. 2000;321(7252):20. http://dx.doi.org/10.1136/bmj.321.7252.20

8. Toledo FG, Taylor A. Alternative site testing at the earlobe tip: reliability of glucose measurements and pain perception. Diabetes Care. 2004;27(2):616-7.

http://dx.doi.org/10.2337/diacare.27.2.616-a
9. Bina DM, Anderson RL, Johnson MS, Bergenstal RM, Kendall DM. Clinical impact of prandial state exercise and site preparation on the equivalence of alternative-site blood glucose testing. Diabetes Care. 2003;26(4):981-5

10. Peled N, Wong D, Gwalani SL. Comparison of glucose levels in capillary blood samples obtained from a variety of body sites. Diabetes Technol Ther. 2002;4(1):35-44. http://dx.doi.org/10.1089/15209150252924067

11. Mira GS, Candido LM, Yale JF. Performance de glicosímetros utilizados no automonitoramento glicêmico de portadores de diabetes mellitus tipo 1. Arq Bras Endocrinol Metab. 2006;50(3):541-9. http://dx.doi.org/10.1590/S0004-27302006000300018

12. Lent R. Cem bilhões de neurônios: conceitos fundamentais de neurociências. São Paulo: Atheneu; 2005.

13. Ferraz DP, Maia FF, Araújo LR. Glicemia capilar em ponta de dedo versus lóbulo de orelha: estudo comparativo dos valores resultantes e preferências dos pacientes. Arq Bras Endocrinol Metab. 2004;48(3):389-93.

http://dx.doi.org/10.1590/S0004-27302004000300010

14. Viana AG, Lins D, Jacob RS, Liberman B. Locais alternativos de coleta de sanguínea na automonitorização da glicemia: um estudo comparativo. Arq Bras Endocrinol Metab. 2003 47(2):S179. 